
entreprise: technologie et temporalité

\title{
La mobilisation numérique de l'immigré tunisien pendant la révolution
}

\section{Feirouz Boudokhane-Lima et Nayra Vacaflor}

\section{OpenEdition}

\section{Journals}

Édition électronique

URL : http://journals.openedition.org/communicationorganisation/4441

DOI : 10.4000/communicationorganisation.4441

ISSN : $1775-3546$

Éditeur

Presses universitaires de Bordeaux

Édition imprimée

Date de publication : 1 décembre 2013

Pagination : 215-226

ISBN : 978-2-86781-878-3

ISSN : $1168-5549$

Référence électronique

Feirouz Boudokhane-Lima et Nayra Vacaflor, « La mobilisation numérique de l'immigré tunisien pendant la révolution », Communication et organisation [En ligne], 44 | 2013, mis en ligne le 01 décembre 2016, consulté le 01 mai 2019. URL : http://journals.openedition.org/ communicationorganisation/4441; DOI : 10.4000/communicationorganisation.4441 


\title{
La mobilisation numérique de l'immigré tunisien pendant la révolution
}

\author{
Feirouz Boudokhone-Lima ${ }^{1}$, Naụra Vacoflor ${ }^{2}$
}

Le déroulement de la révolution tunisienne a mis en évidence le rôle déterminant des médias sociaux dans la mobilisation du public. Le régime de Ben Ali avait la mainmise sur les moyens de communication. À l'image de Big Brother de George Orwell, les photos du président déchu et la couleur mauve, symbole de son parti politique, étaient omniprésentes dans la sphère publique. Face à cette prééminence sémiotique, le citoyen tunisien a pu déployer pendant la révolution une résistance et une créativité à travers les médias sociaux afin de contrarier le discours médiatique dominant. Il était le révolutionnaire et le « journaliste » de sa propre révolution. L'idée était de filmer, de retranscire la réalité à travers des vidéos et de les diffuser via les médias sociaux. On souligne en effet l'émergence d'une production alternative d'informations, et d'autres procédés mis en œuvre, afin de raconter l'actualité.

C'est grâce aux vidéos filmées et postées par leurs concitoyens sur Facebook, que plusieurs Tunisiens résidents en France ont pu rester en lien avec les évènements qui se sont déroulés dans leur pays pendant la révolution. Dans cet article, nous nous pencherons sur la manière dont ces immigrés ont utilisé les réseaux sociaux pendant la " révolution du jasmin ». Nous nous intéresserons à leur perception de Facebook en tant que média alternatif pendant la révolution tunisienne, ainsi qu'à leur mobilisation numérique.

\section{Méthodologie}

Cet article est le fruit d'une enquête qualitative auprès de quinze immigrés tunisiens. Ces personnes ont entre 20 et 50 ans, parmi elles 7

1 Feirouz Boudokhane-Lima est maître de conférences en Sciences de l'Information et de la Communication à l'École Supérieure du Professorat et de l'Éducation de l'Université de Franche-Comté. Elle est membre du laboratoire de recherche C3S (Culture, Sport, Santé, Société). Ses activités de recherche prennent place dans le cadre de la problématique des usages et non-usages des TIC.

2 Nayra Vacaflor est enseignante - chercheuse en Sciences de l'information et de la Communication, membre associée du laboratoire MICA, actuellement chargée de recherche pour le programme MSHA «Citoyenneté en couleurs» Université Bordeaux Montaigne. 
sont de sexe masculin et 8 de sexe féminin. Elles habitent la Communauté Urbaine de Bordeaux (la CUB) et appartiennent à différentes catégories socioprofessionnelles : étudiant, ingénieur, enseignant, employé. Notre étude s'est fondée sur la méthode visuelle du film-élicitation. Selon Marcus Banks, cette méthode de recherche a pour but d'introduire des extraits audiovisuels dans un entretien (Banks, 2001). Nous avons sélectionné 4 vidéos citoyennes représentatives de la révolution tunisienne. L'utilisation de la vidéo permet de libérer la parole relative au sujet traité et de replacer l'interviewé dans le contexte de la révolution. La vidéo joue le rôle d'une fenêtre entre le passé (ce qui a été filmé) et le présent (le moment du visionnement).

\section{Présentation du corpus vidéographique}

Notre corpus comporte 4 vidéos. Ces vidéos ont toutes été extraites de Youtube, mais elles ont été diffusées et partagées sur d'autres réseaux sociaux, notamment Facebook.

Nous allons établir une fiche technique de chaque vidéo, qui comprend les informations suivantes : lien Internet, titre, lieu, durée et un texte descriptif.

\section{Vidéo 1}

Lien Internet : http://www.youtube.com/watch?v=bYaDx-bKJoI

Titre : pain et eau et Ben Ali non : خبز وماء وبن علي لا

Lieu : Tunis, devant le Ministère de l'intérieur, Avenue Habib Bourguiba

Durée : 1 min et 4 sec.

Descriptif : une grande manifestation devant le Ministère de l'intérieur montrant des Tunisiens qui appellent au départ de Ben Ali en criant : "pain et eau et Ben Ali non : خبز وماء وبن علي لا". Dans cette vidéo, on peut voir aussi plusieurs citoyens levant leur téléphone portable, afin de filmer ce moment. On peut déduire ainsi que plusieurs vidéos citoyennes de cette manifestation ont été réalisées et partagées.

\section{Vidéo 2}

Lien Internet : http://www.youtube.com/watch?v=8ZBoHEDKayU\&fea ture=related

Titre : Dégage

Lieu : Tunis devant le Ministère de l'intérieur, Avenue Habib Bourguiba

Durée : 37 secondes

Descriptif : une grande manifestation devant le Ministère de l'intérieur montrant des Tunisiens qui appellent au départ de Ben Ali, la main levée, en huant, et en criant tous ensemble « dégage, dégage ».

\section{Vidéo 3}

Lien Internet : http://www.youtube.com/watch?v=GZPZPzwsRdU\&fea ture=related 
Titre: Tunisie libre, je te mets au défi si tu ne pleures pas :

تونس الحرة أتحداك ان لم تبكي

Lieu : Tunis, Avenue Habib Bourguiba

Durée : $2 \mathrm{~min} 39 \mathrm{sec}$.

Descriptif : la vidéo montre un homme seul ${ }^{3}$, dans l'une des plus grandes artères de Tunis - l'avenue Habib Bourguiba -, annonçant la fuite de Ben Ali en criant haut et fort :

"Ben Ali le chien, n'ayez plus peur, levez vos têtes, n'ayez plus peur, nous nous sommes libérés, le peuple tunisien est libre, le peuple tunisien ne mourra jamais, le grand peuple tunisien, vive la Tunisie libre, gloire aux martyrs, la liberté pour les tunisiens. Vous les tunisiens exilés, qui avez connu l'emprisonnement, ceux qu'on a torturés, ceux qu'on a oppressés, on vous a attristés, on vous a volés, respirez la liberté, le peuple tunisien nous a offert la liberté. Vive le peuple tunisien, vive la grande Tunisie, vive la liberté, gloire aux martyrs, ô tunisiens n'ayez pas peur, le criminel s'est enfui, Ben Ali s'est enfui, Ben Ali a fui le peuple tunisien. Ben Ali s'est enfui, Ben Ali s'est enfui, Ben Ali s'est enfui, Ben Ali s'est enfui, le criminel

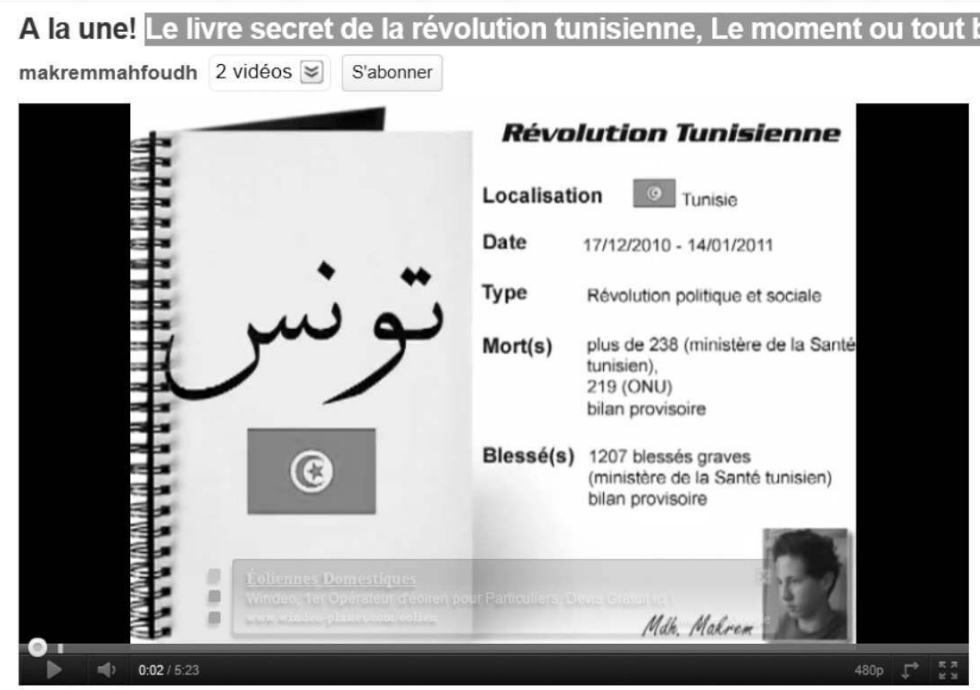

s'est enfui, le voleur s'est enfui, l'assassin s'est enfui, il s'est enfui et c'est le peuple qui commande maintenant, gloire au peuple, gloire aux martyrs, la grandeur pour la Tunisie, et c'est le peuple qui reste. Ô toi mon grand peuple, ô mon cher peuple, toi qui as donné tes enfants, ô toi qui as donné les fruits de tes entrailles, ô toi mon beau peuple, ô toi mon grand peuple, nous nous sommes libérés et Ben Ali s'est enfui, il s'est enfui. La Tunisie maintenant est sans Ben Ali, l'assassin s'est enfui, Ben Ali n'y est plus, le peuple Tunisien nous a débarrassés de Ben Ali, le peuple Tunisien nous a débarrassés de Ben Ali. Moi maintenant je suis dans l'avenue, vive le peuple, vive

3 L'absence de la foule se justifie notamment par le décret d'un couvre-feu et par la terreur semée via des snipers partisans de Ben Ali ; suite à son départ de la Tunisie, il courait ainsi un grand risque. 
la liberté, vive la Tunisie libre, vive notre grand peuple, gloire aux martyrs, votre sang n'a pas coulé pour rien, votre sang n'a pas coulé pour rien. Venez, je suis dans l'avenue ».

\section{Vidéo 4}

Lien Internet : http://www.youtube.com/watch?v=Z5fCgOAa0YY

Titre : Le livre secret de la révolution tunisienne, le moment où tout bascule

Lieu : différents endroits de la Tunisie et à l'étranger

Durée : $5 \mathrm{~min} 23 \mathrm{sec}$.

Descriptif : La vidéo est un montage des moments forts de la révolution tunisienne. Elle commence par une présentation de l'objet de la vidéo, accompagnée de la photo et de la signature du réalisateur. La première séquence montre Mohamed Bouazizi, le jeune vendeur ambulant qui s'est immolé. Les autres séquences sont : des images de différentes manifestations qui ont eu lieu en Tunisie pendant la révolution mais aussi à l'étranger en solidarité avec le peuple tunisien; des images reflétant l'armée dans un rôle de protecteur du peuple de la révolution; d'autres images symboliques et révélatrices de la révolution tunisienne : un manifestant tenant une baguette, comme s'il tenait un fusil ; un char écrasant une photo de Ben Ali; des images de blessés et de morts ; un manifestant porté par la foule tenant une cage ouverte; un militaire donnant une fleur à une petite fille; des images de la police tapant sur les manifestants ; des manifestations dans plusieurs villes tunisiennes et à l'étranger en soutien au peuple tunisien; des drapeaux de pays arabes sous forme de dominos faisant référence à l'effet domino de la révolte dans le monde. Le réalisateur termine son montage par la question suivante : l'hiver des peuples arabes fera-t-il un printemps?

\section{Les réseaux sociaux comme moyens alternatifs}

La contestation numérique à l'encontre du pouvoir tunisien a vu le jour au début des années 2000, via des sites et des blogs collectifs indépendants créés par des cyberactivistes. On cite à titre d'exemples : Takriz, Tunezine, Réveil tunisien, Nawaat et Tunisnews. La cyberdissidence était limitée à certains expatriés et quelques opposants militants sur le territoire tunisien. Du fait de l'interdiction des principaux sites de l'opposition en Tunisie et en exil, leur contenu restait accessible uniquement aux Tunisiens habitant à l'étranger ou ceux qui, vivant en Tunisie, avaient un proxy permettant de contourner la censure.

Dès le début du mouvement révolutionnaire tunisien qui s'est déclenché fin 2010, et pour échapper à la répression et au contrôle de la liberté d'expression, les médias sociaux ont évolué progressivment vers des moyens alternatifs pour mener des actions contestataires. Un nouvel espace médiatique ouvert au débat public commençait à voir le jour. Facebook est alors devenu un pionnier de la protestation en Tunisie. 
Cependant, la blogosphère et les réseaux sociaux ne sont pas les espaces pionniers de la protestation en Tunisie. Les Tunisiens rencontrés soutiennent l'idée que les réseaux sociaux sont devenus progressivement des moyens contestataires alternatifs à des réseaux clandestins. Ils ont constitué un catalyseur qui a appuyé des actions antérieures. Progressivement les réseaux sociaux ont permis le passage d'une contestation déguisée, occultée, à une contestation exprimée, affichée et délocalisée. Les vidéos diffusées sur Facebook, les commentaires, les dénonciations en ligne ont remplacé la diffusion clandestine de tracts et les discussions dissimulées.

"Les réseaux sociaux ont changé la donne » nous dit l'un de nos interlocuteurs dans ce contexte. Il ajoute :

« avant il y avait les tracts, maintenant ça a pris une autre forme : diffusion de vidéos et discussions en ligne, (...), à part mettre ces vidéos sur Facebook ou autres, les tunisiens ne pouvaient rien faire de ça, si tu les prends avec toi sur CD pour voyager, à la douane tu es fouillé, pareil le courrier est fouillé, tout était contrôlé ! Tu vois ce livre là j'avais peur de voyager avec, j'étais obligé de le scanner, de le mettre sur une clé USB, de changer son titre, de changer l'extension au cas où ils fouillent mon ordinateur, ils n'allaient pas le trouver mais j'avais peur, on a chopé un médecin qui est rentré avec ça, il a pris 6 mois de prison ».

Plusieurs Tunisiens élaboraient en effet diverses tactiques pour contourner la censure et rompre le monopole de l'information étatique.

Nos interlocuteurs nous ont fait part de leur crainte, avant la période de la révolution, de la répression et du contrôle que le pouvoir de Ben Ali avait sur les moyens de communication. Pour parler au téléphone avec leur famille de la situation en Tunisie, des codes étaient souvent utilisés pour ne pas nommer le président et sa femme, nous expliquent certains Tunisiens rencontrés.

Le contrôle des réseaux de communication était un enjeu de pouvoir et de survie pour le régime totalitaire et répressif de Ben Ali. Les meilleurs ingénieurs en informatique tunisiens étaient recrutés par le Ministère de l'intérieur. Le régime avait la mainmise sur l'ATI (Agence Tunisienne d'Internet), l'unique point d'accès. Dès le début du mouvement contestataire, tous les réseaux sociaux ont été censurés en Tunisie, remarquent nos interlocuteurs, seul Facebook étant épargné : "la censure était totale, mais quand les critiques notamment de l'étranger ont commencé à surgir, le régime devait faire un choix, il a levé le verrou sur Facebook, et a censuré tout le reste : youtube, dailymotion, mais il n'a pas vu le poids de Facebook »(Anis, ingénieur).

Les Tunisiens rencontrés comparent le soulèvement de 2011 à la grande vague de protestations populaires qui a secoué en 2008 la région de Gafsa (sud-ouest de la Tunisie) et plus particulièrement les villes du bassin minier dont celle d'Errdaif. Ces protestations, nous explique Anis, l'un des interrogés, sont presque passées inaperçues. Il dit : 
«à l'époque Facebook n'était pas aussi généralisé, pourtant le soulement d'Errdaif était très violent. C'est en faisant ce genre de parallèle qu'on se rend compte finalement que Facebook a clairement été un catalyseur pendant la révolution. Facebook te met en contact avec 300 voir 400 personnes qui peuvent voir tout de suite ce que tu partages, c'est quelque chose de très, très puissant, la diffusion peut se faire très, très vite ».

Même si la contestation numérique s'appuie sur des engagements et d'autres formes de mobilisation prélables, « les capacités offertes par le réseaux permettent une plus grande diversité et une plus large distribution des causes et des publics mobilisés » (Cardon, 2010, p. 127).

Pour nos interlocuteurs, l'information concernant les abus de l'ancien régime provenait essentiellement d'Internet. Pendant la révolution, Facebook est devenu un moyen alternatif d'information qui leur a permis de rester en lien avec la Tunisie. Les vidéos filmées et postées sur Facebook par leurs concitoyens en Tunisie représentaient un témoignage vivant et authentique d'une situation que les médias traditionnels ont trop tardé à couvrir. Tous nos interlocuteurs nous ont fait part de leur perte de confiance dans les médias tunisiens. Les extraits d'entretiens qui suivent en témoignent : «Ces vidéos citoyennes que vous nous montrez ont les a vues, revues, partagées et repartagées sur Facebook. Elles inspirent de la crédibilité, ce n'est pas le camouflage et le truquage de la chaîne nationale tunisienne » (Sounya, étudiante) ; "Pendant que les gens se faisaient descendre par balles dans les manifs, la chaîne tunisienne passait des chansons, des documentaires sur la mer, nos confrères là-bas étaient bien obligés de montrer ce qui se passait dans la rue, il n'y avait que Facebook pour diffuser, partager ces vidéos et montrer la réalité » (Aïcha, étudiante); " pour moi ces vidéos sont devenues comme la marque de fabrique de notre révolution, ce sont des vidéos qui viennent du cœur de l'événement et pas des vidéos qui sont faites avec des mises en scène et des trucages, comme nous a habitués la chaîne tunisienne » (Sonia, étudiante).

Nos interlocuteurs insistent tous sur cette idée que les vidéos citoyennes de la révolution diffusées sur Facebook étaient reprises par les chaînes de télévision telles qu'Aljazira et France 24. Ils considèrent en effet que leurs concitoyens, livrés à eux-mêmes, étaient les acteurs et les reporters de cette révolution. Le Tunisien était passé du rôle de simple récepteur d'une information manipulée à celui d'émetteur, de journaliste citoyen jouant un rôle actif dans les processus de récupération, de reportage et d'analyse de l'actualité (Bowman et Willis, 2003).

\section{La mobilisation numérique}

«Internet accueillit (...) de nouvelles formes de mobilisation informationnelle qui se caractérisent par leur spontanéité ainsi que leur nature auto-organisée et décentralisée »(Cardon, 2010, p. 118). De nombreux 
exemples de mobilisations récentes se sont déroulées via les réseaux sociaux. Quand des milliers de manifestants moldaves ont protesté dans les rues contre leur gouvernement en 2009, leur mouvement a été appelé « la Révolution Twitter ", en raison de l'outil de communication utilisé. Il en était de même pour les contestations contre le régime iranien la même année. La révolution tunisienne s'est déroulée selon un tel processus. Par ailleurs, nos interlocuteurs considèrent que le réseau social catalyseur de cette révolution, était Facebook.

Les Tunisiens rencontrés nous ont parlé de " leur révolution parallèle ». Ils refusent d'être considérés comme des spectateurs. Ils considérent que leur action s'intègre dans un mouvement révolutionnaire participatif. Nous empruntons le terme de «spect-acteur » à Augusto Boal (Boal, 1996), afin de montrer que ces immigrés n'étaient pas de simples spectateurs, mais des « acteurs actifs » de la révolution. Cela veut dire que leurs actions ne se résumaient pas à celles de visionner et de partager des vidéos, mais surtout de commenter, de débattre, de dénoncer, de mobiliser et de militer :

"Au début du déclenchement des protestations, il y avait des gens en Tunisie qui n'en parlaient même pas, pour eux c'était un événement banal qui allait s'arrêter comme à chaque fois, et moi curieusement cette fois j'y croyais et je n'ai pas arrêté de sensibiliser, de motiver mes copains là-bas. Je sentais que c'était de mon devoir de dénoncer ce qui se passait et d'encourager en quelque sorte le processus révolutionnaire » (Hana, étudiante);

"Pour moi tout ce que j’ai pu écrire et dénoncer sur Facebook, c'était très clairement ma part de participation à cette révolution. C'était le minimum qu'on pouvait faire, on était ici, il fallait au moins se mobiliser par la parole, par l'expression, par le fait de passer l'information. Enfin moi j'ai essayé d'expliquer à certains amis ce qui se passait parce qu'ils y en avaient qui s'en foutaient complètement au début et c'était vraiment le moment de mobiliser, parce qu'il fallait que tout le monde bouge !» (Safa, étudiante).

Nos interlocuteurs nous ont parlé d'autres formes d'actions de mobilisation numérique via Facebook, comme l'échange de proxys pour contourner la censure et la diffusion d'informations de sites d'opposants interdits en Tunisie. Les extraits d'entretiens qui suivent en témoignent : "Il y avait beaucoup de blogs qui étaient censurés en Tunisie et nous ici on avait accès aux informations qui circulaient sur ces blogs. On allait sur les sites d'opposition, on prenait les informations et on les postait sur Facebook » (Hassan, employé) ;

«Ce que j’ai pu faire aussi c'est les échanges des proxys avec des hackers pour contourner le contrôle, j’ai essayé de les partager, de les poster. Parfois tu tombes sur un proxy qui marche et au bout de deux jours il ne marche plus, parce qu'ils ont les moyens là-bas de les détecter, donc il fallait à chaque fois en trouver d'autres » (Mokhtar, enseignant).

Jacques Attali (2003) proposait l'hypothèse de la résurgence de formes particulières de nomadisme et de nouvelles mobilités liées à l'émergence des 
technologies. Omniprésents pendant la révolution tunisienne en termes de possibilités d'accès via Internet (en l'occurrence Facebook), les enregistrements audiovisuels à travers les téléphones mobiles ont permis effectivement de se connecter, voire « d'être » connecté en quasi-permanence, dans les deux pays. La mobilisation numérique pendant la révolution a permis l'émergence de trois pratiques : l'expression audiovisuelle numérique sur Internet, les transferts de données (partage permanent) et la mobilisation des personnes à l'étranger (de manière virtuelle et physique). Navigation, transfert et mobilisation désignent trois réalités faisant référence à une dimension symbolique de « la portabilité » des images retraçant la révolution. L'association de la portabilité des outils numériques, de l'interopérabilité du réseau Facebook et des fenêtres sensorielles ont favorisé une " connexion communautaire ", où le « spectacle " multimédia a pris autant d'importance que la relation à autrui.

Nos interlocuteurs ont par ailleurs souligné l'engagement différent qui était le leur vis-à-vis de leurs confrères en Tunisie, les tensions et la crainte diminuant avec l'éloignement. Les propos suivants sont révélateurs de cette idée :

«Au début du mouvement, les gens là-bas avaient peur de s'exprimer sur Facebook. Au début ils partageaient des vidéos mais sans prise de position, sans réaction, ni dénonciation. Je pense qu'on avait moins peur qu'eux, en étant ici on risquait moins les représailles. Je me rappelle j’ai insulté le pouvoir sur Facebook, je n'ai pas arrêté de dénoncer, de motiver mes amis. J'incitais à la révolte en quelque sorte. J'avais la rage et je n'avais que les mots pour mobiliser » (Nizar, employé);

«Les gens ici avaient moins peur de dénoncer, ils n'avaient rien à craindre, bon au pire ils ne pouvaient plus rentrer au pays si Ben Ali restait au pourvoir. Moi mes parents avaient peur pour moi, ils me mettaient en garde, ils me disaient d'arrêter. Pour moi il était bien sûr hors de question de faire marche arrière "(Hana, étudiante).

La réflexion de Dominique Cardon (2010) au sujet des réseaux sociaux et de cette nouvelle identité permettant à des internautes, majoritairement jeunes et jusque-là faiblement politisés, de pouvoir s'exprimer et peut-être venir grossir les rangs des manifestants, semble intéressante. Il explique ainsi l'aspect catalyseur de Facebook, lors de la révolution tunisienne :

« on est dans le clair-obscur, on n'a pas l'impression de parler dans l'espace public. Et voilà que les conversations amicales se débrident et qu'on se retrouve à dire ce qu'on pense de Ben Ali. Quand les proches disent "Tu as raison", on assiste à un renforcement du pouvoir de la parole qui, partant de petites niches individuelles, gagne en puissance jusqu'à exprimer une indignation. C'est complètement explosif ! (Cardon, 2011, [En ligne]).

« La démocratie se mesure à la capacité expressive des citoyens (...). Le propre du Web, c'est l'auto-organisation, la création d'espaces à soi ", rajoute Cardon (Ibid.). Le réseau Facebook semble être en affinité avec les nouvelles formes d'organisation et de mobilisation. 
La communauté qui s'est établie à partir de la mobilisation numérique, s'est installée et a trouvé un lieu précis : celui d'un espace permettant l'expression libre et engagée. Elle est devenue un acteur révolutionnaire de lutte et de contre-pouvoir, à la recherche d'une histoire, de faits et d'une longévité commune (Maffesoli, 1988). Cette communauté mobilisée était composée de personnes qui avaient en commun des valeurs, des croyances, des intérêts et des ambitions en vue de l'amélioration des conditions de vie dans leur pays. Elle était caractérisée par une identité collective numérique et parfois " physique », comprenant des actions sur le terrain. En effet, outre l'engagement numérique de nos interlocuteurs, ces derniers ont souligné leurs actions de rassemblement, de sensibilisation et de manifestations dans les rues bordelaises et dans les locaux de l'ATF Bordeaux (Association des Tunisiens en France). Les Tunisiens immigrés se sont impliqués non seulement dans une démarche d'engagement virtuel, mais aussi dans une démarche pragmatique, afin de réaliser " leur contribution à la révolution » en étant en France. Ils ont joué le rôle de « spect-acteurs » qui se sont engagés dans la création d'un espace d'information et de communication « libres ». Nous avons en effet recensé les différentes «spect-actions " menées sous trois rubriques :

\begin{tabular}{|l|l|l|}
\hline $\begin{array}{c}\text { Communication } \\
\text { et « data-dump } \\
\text { révolutionnaire }\end{array}$ & $\begin{array}{c}\text { Circulation et échanges } \\
\text { numériques }\end{array}$ & \multicolumn{1}{|c|}{$\begin{array}{c}\text { Création et durabilité } \\
\text { révolutionnaire }\end{array}$} \\
\hline $\begin{array}{l}\text { Manifestations dans la } \\
\text { rue. }\end{array}$ & $\begin{array}{l}\text { Communication, échanges } \\
\text { (parler de cela aux } \\
\text { "autres "). }\end{array}$ & $\begin{array}{l}\text { Création d'évènements } \\
\text { et de groupes " pro- } \\
\text { révolution » via Facebook. }\end{array}$ \\
\hline $\begin{array}{l}\text { Rédaction d'articles } \\
\text { (journalistiques, de } \\
\text { réflexion, de recherche, } \\
\text { création de blogs.) }\end{array}$ & $\begin{array}{l}\text { Réunion « physique » } \\
\text { entre Tunisiens pour } \\
\text { suivre les événements en } \\
\text { Tunisie. }\end{array}$ & $\begin{array}{l}\text { Voyages en Tunisie, } \\
\text { déplacements sur les lieux } \\
\text { de l'évènement. }\end{array}$ \\
\hline
\end{tabular}

Nous soutenons l'idée que les immigrés tunisiens ont accordé un sens à leurs actions numériques. Ces dernières peuvent être lues « [...] à la fois comme des processus sociaux et comme un champ de bataille » (Martin-Barbero, 2002, p. 10). Les significations et les valeurs qui se sont formées entre ces individus se fondent sur « la base de leurs relations, de leurs conditions historiques, de leurs pratiques et traditions vécues " (Hall, 2007, p. 43), ainsi que sur leur mobilisation contre un régime dictatorial.

Lidée de centrer une action numérique dans une révolution a joué selon Nader Abid et al. (2011) un rôle " empathique et solidaire " avec les

4 Il était important pour nos interviewés que chaque immigré tunisien pût créer, trouver des documents et les mettre en ligne, afin qu'un large public puisse y avoir accès, les explorer et en identifier la pertinence. 
manifestants. «Le dispositif (Facebook) est devenu un espace d'échange et de discussion autour de thématiques politiques et idéologiques " (Abid et al., 2011, p. 229). Les évènements de la révolution ont pris une place importante dans le vécu des immigrés tunisiens en France. L'usage de Facebook comme outil d'engagement et de mobilisation était devenu une pratique omniprésente : «je me rappelle, je ne dormais presque plus, j'étais 22 heures sur 24 sur Facebook, même au travail! Il fallait que je sois connecté, que je sois dans la révolution et dans la mobilisation " (Anis, ingénieur). Une nouvelle conception de l'espace public a émergé. Pour le Tunisien "déterritorialisé ", il s'agit d'un espace où la prise de parole n'est plus réservée mais libre. «C'est en tout cas le premier gain de cette révolution, en attendant que les autres revendications soient acquises ", nous dit enfin l'un de nos interlocuteurs.

\section{Conclusion}

Le désir d'une vie meilleure où liberté, dignité et justice sociale sont les droits fondamentaux de chacun a été l'objectif poursuivi par la révolution tunisienne. Les dynamiques préalables, les jugements sociétaux, l'enfermement de la liberté de parole, sont autant d'éléments qui ont perduré dans « l'être " $\mathrm{du}$ Tunisien avant, lors de et après la révolution. Fabien Granjon souligne que «le réseau des réseaux peut être un fabuleux catalyseur, mais n'est certainement pas la cause, ni même l'élément principal de l'action collective » (Granjon, 2011, [En ligne]). Nos analyses montrent que Facebook a constitué un outil important en vue de la mobilisation et de l'engagement de nos interlocuteurs. $\mathrm{La}$ « révolution de jasmin » n'est certes pas l'œuvre de ce réseau social. Mais nos interlocuteurs, étant à l'étranger, ont insisté sur l'idée qu'ils n'auraient pas pu imaginer leur « révolution parallèle » sans cet outil. Le caractère immédiat des échanges informationnels et de la mobilisation sur Facebook ont tenu une place déterminante dans leur vécu numérique et réel au cours de cette période.

\section{BIBLIOGRAPHIE}

ABID N. et al. « Le rôle des médias et des TIC dans les révolutions arabes : l'exemple de la Tunisie ", Chimères, 2011/1, n 75, p. 219-235.

ATTALI J., L'Homme nomade, Paris, Fayard, 2003.

BANKS M., Visual Methods in Social Research, London, Sage, 2001.

BOAL A., Théâtre de l'opprimé, Paris, La Découverte, 1996.

BOWMAN S., WILLIS C., We Media : How audiences are shaping the future of news and information, 2003 [En ligne, page consultée le 12 mars 2013]. Disponible sur : http:// www.hypergene.net/wemedia/download/we_media.pdf

CARDON D., "Plus qu'un mouvement social, des mouvements d'individus. Entretien avec Amaury de Rochegonde », Stratégies, n 1659, 15 novembre 2011 [En ligne, page consultée le 22 février 2013]. 
Disponible sur : http://www.strategies.fr/actualites/medias/177609W/dominiquecardon-plus-qu-un-mouvement-social-des-mouvements-d-individus.html

CARDON D., GRANJON F., Médiactivistes, Paris, Les Presses de Sciences Po, 2010.

CARDON D., La démocratie Internet, promesses et limite, Paris, Seuil, 2010.

FERRON B., "Les médias alternatifs : entre lutte de définition et lutte de délégitimation ", Les enjeux de l'information et de la communication, 2006 [En ligne , page consultée le 16 février 2013]. Disponible sur : http://w3.u-grenoble3.fr/les_enjeux/2006supplement/Ferron/

FILIU J.-P., La Révolution arabe : Dix leçons sur le soulèvement démocratique. Paris, Fayard, 2011.

GRANJON F., «Le web fait-il les révolutions ? », Sciences Humaines, n² 229, 20 juillet, 2011.

[En ligne, page consultée le 20 janvier 2013]. Disponible sur : http://www.cairn.info/ article_p.php?ID_ARTICLE=SH_229_0013

GRANJON F., LE FOULGOC A., «Des usages sociaux de l'actualité. Les expériences médiatiques des publics internautes ", Réseaux, n 28, 2010, p. 225-253.

HALL S., Identités et Cultures. Politiques des cultural studies, Édition établie par Maxime Cervulle, Paris, Éditions Amsterdam, 2007.

JAURÉGUIBERRY F., " Le moi, le soi et Internet », Sociologie et Société, vol. 32, n 2, 2000, p. $135-152$.

MAFFESOLI M., Le temps des tribus. Le déclin de l'individualisme dans la société de masse, Paris, Méridiens Klincksieck,1988.

MARTIN-BARBERO J., Des médias aux médiations, Paris, CNRS, 2002.

MIÈGE B., L'espace public contemporain : approche info-communicationnelle, Grenoble, PUG, 2010.

MONGEAU O., "La révolte des indignés de la Toile », Stratégies, n 1659, 15 décembre 2011 [En ligne , page consultée le 18 janvier 2013]. Disponible sur : http:// www.strategies.fr/actualites/medias/177465W/la-revolte-des-indignes-de-la-toile.html

Résumé : La révolution tunisienne a suscité beaucoup de réflexions et d'analyses quant à l'utilisation des réseaux sociaux et d'Internet pendant son déroulement. Notre article propose un regard particulier sur l'immigré tunisien en France et son engagement militant à travers la plateforme Facebook. Cette dernière est présentée comme un outil de communication alternatif qui a contribué à la mobilisation numérique des immigrés. Facebook a laissé place à un nouvel espace de confrontation et de libération de la parole. Dans ses formes les plus apparentes, cet outil a nourri la mobilité «physique » nécessaire dans les domaines traditionnels d'expression révolutionnaire. Avec cette recherche, nous proposons un renouvellement de l'adoption « communicationnelle » dans l'espace public en offrant une nouvelle perspective des usages numériques en tant qu'outils d'action.

Mots-clés : Révolution tunisienne, immigré tunisien, Facebook, mobilisation numérique, spect-acteur. 
Abstract : The Tunisian revolution provoked many reflections and analysis on the use of social networks and the Internet during this revolution. Our article focuses on the Tunisian immigrant population in France and their commitment to the militants through Facebook. This social network is presented as an alternative communication tool wich contributed to the digital mobilization of these immigrants. Facebook became a new space of confrontation and liberation of the word. This tool fuelled the necessary "physical " mobility in the traditional domains of revolutionary expression. With this research, we propose a review on communication adopted in the public place by offering a new perspective on digital practices as tools of action.

Keywords : Tunisian revolution, Tunisian immigrant, Facebook, digital mobilization, spect-actor. 